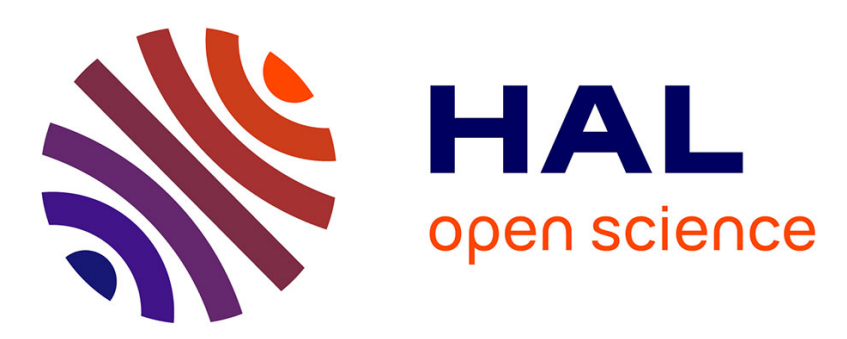

\title{
Le " numérique éducatif " à l'épreuve des pratiques scolaires : petits arrangements avec le marché
}

\author{
Karine Aillerie
}

\section{To cite this version:}

Karine Aillerie. Le " numérique éducatif " à l'épreuve des pratiques scolaires : petits arrangements avec le marché. Hermès, La Revue - Cognition, communication, politique, 2017, 10.3917/herm.078.0023 . halshs-02165597

\section{HAL Id: halshs-02165597 https://shs.hal.science/halshs-02165597}

Submitted on 26 Jun 2019

HAL is a multi-disciplinary open access archive for the deposit and dissemination of scientific research documents, whether they are published or not. The documents may come from teaching and research institutions in France or abroad, or from public or private research centers.
L'archive ouverte pluridisciplinaire HAL, est destinée au dépôt et à la diffusion de documents scientifiques de niveau recherche, publiés ou non, émanant des établissements d'enseignement et de recherche français ou étrangers, des laboratoires publics ou privés. 
Aillerie K. (2017). « Le « numérique éducatif » à l'épreuve des pratiques scolaires : petits arrangements avec le marché », Hermès, 78. Les élèves entre cahiers et claviers. pp.23-30

\section{Le « numérique éducatif » à l'épreuve des pratiques scolaires : petits arrangements avec le marché}

Indépendamment d'injonctions ministérielles récurrentes, des usages des technologies se construisent à l'école, au fur et à mesure des opportunités et des besoins quotidiens des acteurs, enseignants et élèves. Ce « fait numérique » a ainsi été récemment décrit pour ce qui concerne le lycée par Cottier et Burban (2016). Le lycée n'est pas seul concerné par cette problématique des pratiques installées mais il constitue un terrain d'observation particulièrement intéressant du fait de l'âge des élèves et de par l'existence à ce niveau de situations pédagogiques possiblement propices au déploiement de parti pris pédagogiques intégrant les technologies. Notre propos s'appuie sur une étude ${ }^{1}$, réalisée dans un lycée porteur d'un projet numérique équipant élèves et enseignants de dispositifs numériques individuels nomades et mettant à leur disposition des infrastructures adéquates (Wifi, ENT, réseau, serveurs de stockage, etc.). La spécificité de ce terrain nous permet ainsi d'écarter les problématiques d'ordre logistique et d'interroger directement «l'épaisseur » des pratiques (Jeanneret et al., 2003). Le choix d'une approche basée sur la translittératie, dont nous décrirons le potentiel épistémologique et méthodologique, nous permet d'interroger la primauté accordée ou non au numérique, la possible continuité entre la classe et ce qui se passe en dehors, les outils mobilisés. A ce titre, dans le contexte socio-économique actuel et à la lumière des ambitions institutionnelles affichées, nous posons la question des moyens réellement utilisés par les élèves pour permettre le travail collectif et la prolongation des tâches de recherche d'information ou d'organisation en dehors des heures imparties : quelle est la place des outils mis en place par l'établissement ? Quelle concurrence des outils du marché (GAFAM), plébiscités au quotidien par les usagers?

\section{« Le » numérique au centre de la politique éducative}

L'objectif actuel de l'institution scolaire est de «faire entrer l'École dans l'ère du numérique », c'est-à-dire de profiter des «opportunités offertes» par «le numérique», de former les personnes et d'équiper les établissements en matériels et ressources. Un rôle déterminant y est attribué aux environnements numériques de travail (ENT), comme «prolongement numérique de l'établissement » : pour non seulement organiser la vie scolaire mais pour centraliser l'accès aux ressources et la communication, pour soutenir le « travail collaboratif ». Ce parti pris est présenté comme cohérent avec le choix d'équipements mobiles (tablettes) : | «afin d'instaurer une continuité des usages, dans la classe et à la maison »². Cette politique poursuit de précédents programmes d'introduction de l'informatique ou des TICe (technologies de l'information et de la communication pour l'enseignement) à l'école. Notons, cette fois, le choix du substantif «numérique » ou de

\footnotetext{
${ }^{1}$ Ces travaux sont issus du projet ANR Translit (2013-2016), portant sur les visages possibles de la translittératie en action au sein de situations scolaires et non scolaires.

| ${ }^{2}$ http://ecolenumerique.education.gouv.fr/plan-numerique-pour-l-education/
} 
l'expression «numérique éducatif», terminologie en tant que telle significative d'un rapprochement systématique entre mise à disposition de matériels et transformation subséquente des pratiques d'enseignement et des apprentissages. Cette relation de causalité est cependant loin d'être évidente (Amadieu \& Tricot, 2014). Pour ce qui regarde les compétences des enseignants, trois axes majoritaires sont déclinés : la maîtrise des outils, les usages disciplinaires et les méthodes d'enseignement, la culture numérique et l'éducation aux médias et à l'information $(\mathrm{EMI})^{3}$. Pour ce qui concerne les élèves, les compétences exigées sont relatives à la créativité, la pensée critique, la résolution de problèmes et la capacité à produire des contenus pertinents et de qualité (voir par exemple les items du B2i niveau lycée : travailler dans un environnement numérique évolutif; être responsable; produire, traiter, exploiter et diffuser des documents numériques; organiser la recherche d'informations ; communiquer, travailler en réseau et collaborer). Travail collectif, démarche de projet, production, recherche d'information au moyen des différents médias et outils de recherche à disposition, ces items se retrouvent en partie dans les critères d'évaluation des TPE.

De cette contextualisation, retenons la centration sur «le numérique » et l'accent porté sur les équipements et les ressources, au service d'une pédagogie collaborative, interdisciplinaire et transversale. Retenons également la vision d'une porosité certaine entre les contextes sociaux et académiques: les usages quotidiens, des jeunes en particulier, justifiant la mise en place de cette stratégie institutionnelle ${ }^{4}$.

\section{Un contexte privilégié pour réinterroger les pratiques de terrain}

L'investigation qui fait la base de cette contribution s'est déroulée au sein d'un lycée pilote et lors d'activités pédagogiques spécifiques (Modules InterDisciplinaires ou MID) en seconde. Depuis 2013, un projet à l'échelle de l'établissement a été mis en œuvre : enseignants et élèves disposant d'appareils individuels (tablettes ou ordinateurs portables). Les compétences des élèves y sont évaluées et répertoriées via un webfolio $^{5}$. Le projet pédagogique de cet établissement est historiquement centré sur le développement de l'autonomie des élèves et sur l'intégration des technologies dans les pratiques disciplinaires et interdisciplinaires. Des activités spécifiques y sont ainsi menées, similaires aux TPE, basées sur les travaux de groupes et la pédagogie par projet. Pour cette étude, deux modules (une session de 3 heures par semaine sur un semestre) et deux classes ont été observés, sur la base d'une approche ethno-méthodologique de la cognition située et distribuée (Conein, 2004) prenant en compte les pratiques réelles des acteurs et leurs représentations. Concrètement, les élèves devaient, par groupes de 3 ou 4, produire dans un cas un blog et une vidéo, dans l'autre deux vidéos dont un «pocket film », court clip vidéo pris avec un smartphone, une tablette ou un appareil photo compact. Cette situation s'avère en partie correspondre aux ambitions nationales. C'est à ce titre qu'il nous paraît intéressant d'y confronter des interrogations assez classiques

\footnotetext{
${ }^{3}$ https://www.legifrance.gouv.fr/eli/arrete/2013/7/1/MENE1315928A/jo/texte

${ }_{4}$ http://www.huffingtonpost.fr/najat-vallaudbelkacem/le-numerique-a-lecole-un-investissement-davenir

5 Portfolio numérique au sein duquel sont compilés pour chaque élève les travaux individuels et collectifs menés au cours de la scolarité, les compétences acquises, les participations et expériences menées dans le cadre scolaire, périscolaire et extrascolaire.
} 
quant aux usages des technologies à l'école. Que se passe-t-il en effet, dès lors que l'équipement est massif, l'accès prévu aux ressources et espaces de stockage, l'organisation pédagogique adaptée ? Nous ne prenons évidemment pas pour acquis que la mise à disposition détermine les usages. Mais il nous semble bien que l'interrogation d'un lieu privilégié de ce point de vue peut autoriser une prise de recul et permettre de requestionner des situations standards. Comment s'ordonnent dès lors les pratiques réelles des acteurs, élèves et enseignants, à cette aune ?

\section{Le choix d'une perspective translittératique}

Notre approche théorique est celle de la translittératie définie comme « la capacité à lire, écrire et interagir à travers un éventail de plateformes, d'outils et de medias, des signes à l'oralité en passant par l'écriture manuscrite, l'imprimé, la télévision, la radio et le cinéma, jusqu'aux réseaux socio-numériques » (Thomas et al, 2007). Cette définition fondatrice a été depuis largement amendée (Ipri, 2010 ; Delamotte, Liquète \& Frau-Meigs, 2014 ; Sukovic, 2017).

C'est le préfixe «trans » qui fait la singularité de l'approche translittératique au sein des réflexions sur la notion de littératie. Trois axes d'acception peuvent être ici dégagés (Liquète, 2012). La dimension la plus explicite concerne l'interaction entre les différentes littératies médiatiques et la capacité de passer de l'une à l'autre. En cela, la perspective translittératique nous est utile pour sortir de la seule entrée «numérique » gommant les complexes articulations entre technologies émergentes et médias préexistants.

Le préfixe «trans » fait également référence aux connexions éventuellement possibles entre différents contextes, ici entre l'espace-temps de la classe et le hors classe ou hors école. Cette mobilité symbolique s'énonce en termes d'inégalités. La recherche a ainsi déjà montré qu'une forte hétérogénéité caractérise les usages numériques adolescents: certains jeunes parvenant à rentabiliser scolairement leurs pratiques quotidiennes tandis que d'autres ont des habitudes difficilement compatibles avec les exigences académiques (e.g. Robinson, 2015). Concernant les enseignants, leurs pratiques numériques personnelles n'augurent pas non plus systématiquement de pratiques en classe comparables ${ }^{6}$. A ce titre, la perspective translittératique nous permet d'interroger les connexions et les hiatus potentiels entre la classe et ce qui se passe en dehors.

Enfin, le préfixe «trans » met également en évidence la nature collective des pratiques en contexte. Cette dimension est cruciale à l'heure où le Web est social (réseaux sociaux, commentaires, folksonomies, etc.) et où les activités scolaires impliquant le recours aux technologies sont pensées sur la base du travail collectif (Leeder \& Shah, 2016).

\section{La diversité revendiquée des supports et sources d'information}

Soulignons d'emblée la dimension multi médiatique des situations observées. En effet, malgré l'importance accordée à la technologie numérique dans cet établissement, de multiples médias sont physiquement et

\footnotetext{
${ }^{6}$ https://cache.media.eduscol.education.fr/file/ETIC_et_PROFETIC/15/4/PROFETIC_2016_-_Rapport_complet_648154.pdf
} 
symboliquement présents en classe. Les bureaux des élèves sont encombrés de notes papier (par ailleurs souvent photographiées au moyen des téléphones portables), de livres, de journaux etc., cohabitant avec les favoris de sites Web, les documents d'organisation sur Google Docs, les conversations de travail sur Facebook, etc. Sont sollicités les ordinateurs fixes du centre de documentation en même temps que les appareils mobiles scolaires et/ou personnels des élèves (tablettes, téléphones, baladeurs). Les enseignants interrogés acceptent les dispositifs personnels des élèves en classe, au début et parfois encore à contrecœur : c'est au minimum un fait social qu'il faut accepter ou bien un défi pédagogique à relever.
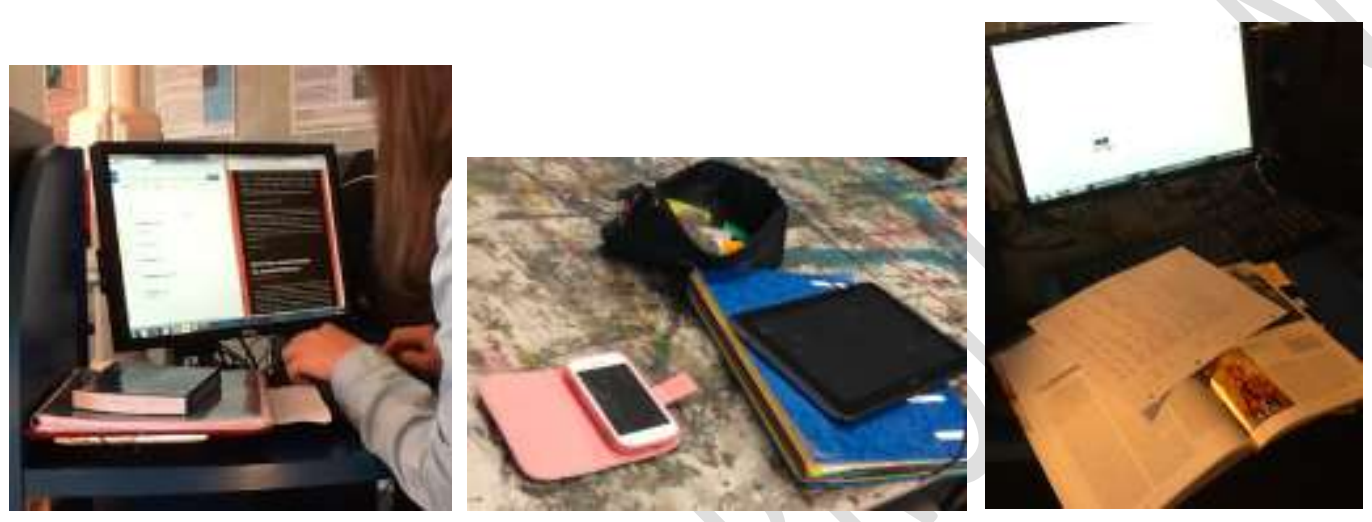

Symboliquement, cette diversité est également évidente dans les exigences scolaires. Tant dans les consignes que dans les critères d'évaluation, les enseignants interrogés insistent en effet sur l'importance d'utiliser plusieurs supports. Cet objectif d'apprentissage est présenté comme une habitude que les élèves n'ont pas, une compétence à acquérir. Les enseignants insistent sur le fait que le travail des élèves doit être basé sur un éventail médiatique aussi large que possible afin qu'ils «(...) ne se focalisent pas sur un seul type de support. Parce que, tout simplement, c'est trop facile. Ils ont tous tendance sinon, à privilégier simplement le support Internet et je pense sincèrement que ce n'est pas la solution. Notre rôle c'est aussi d'élargir leur vision à ce niveau-là et qu'ils puissent toucher un petit peu à tout ». Ainsi, au cours des situations observées, les enseignants relancent continuellement les élèves, se souciant de la qualité documentaire autant que de la diversité des ressources mobilisées : «combien de références magazines?»; «combien de références livres ?»; «Pensez aux ressources audiovisuelles » etc. Il est à noter que l'étape de recherche, de recensement des informations et des sources utiles au projet, est conçue et menée dans une perspective proprement collaborative, tous les membres des groupes contribuant en même temps à alimenter la bibliographie.

\section{La prolongation des organisations collectives en dehors des temps formels d'apprentissage}

L'un des aspects constitutif de l'activité observée est le travail de groupe, désigné dans les documents du lycée comme «collaboration » au sens général de ce terme. Les élèves doivent constituer les groupes par 
eux-mêmes, sur la base de l'intérêt pour le thème à traiter, tenant compte de la mixité des genres et de certaines associations contreproductives. Ils se regroupent le plus souvent par affinités et selon les habitudes déjà prises de travailler ensemble : la complémentarité des compétences est ici souvent recherchée. Ils décident également de l'organisation interne au groupe et des outils qui leur seront utiles. Ainsi, un enseignant précise : «On les laisse libre, parce que je pense que c'est à eux de s'organiser et... bon, on peut parfois leur donner quelques recommandations mais globalement, c'est eux qui ont créé leurs espaces, ils se sont débrouillés ». Obligation leur est faite cependant de recourir régulièrement au réseau informatique du lycée, en cas d'absence et pour que le travail soit vu par les enseignants. Cet impératif fait partie des critères d'évaluation et les répertoires sont ainsi organisés en fonction de l'avancement des travaux (documents supports, travaux en cours, travaux à évaluer, travaux finis). Mais, si l'infrastructure informatique du lycée est au service des objectifs d'apprentissage, nous verrons qu'elle n'occupe qu'une partie des usages et que les élèves sollicitent les outils du marché pour s'organiser.

Si les enseignants interrogés évoquent les exigences parfois importantes qui sont imposées aux élèves, ils déclarent faire en sorte que le temps de travail imparti soit suffisant pour y répondre. Observations et entretiens montrent cependant que la plupart des groupes a travaillé en dehors. Pour les enseignants, ceci relève soit d'un problème d'organisation, soit est quelque chose d'inévitable que les élèves doivent finalement apprendre à gérer. C'est la réalisation de la production finale qui est concernée par ce temps de travail à l'extérieur de la classe voire du lycée, mais également et surtout l'organisation au sein du groupe. S'organiser collectivement, y compris pendant les pauses, le temps personnel ou même les vacances, s'avère parfois compliqué voire stressant. Les élèves évoquent les nombreux problèmes auxquels ils doivent faire face (manque de compétences, perte de fichiers, incompatibilités techniques etc.) et les éléments mobilisés pour y répondre (leur expérience personnelle, l'équipement domestique, la capacité de leurs parents ou amis pour les aider, par exemple). Ils pointent particulièrement le temps qui leur a manqué quant à la dimension métacognitive et concrète de l'organisation collective (se répartir les rôles, planifier le travail, décider des outils pertinents) : «(...) en fait le numérique ça met quand même un certain temps à se mettre en place et une fois que c'est mis en place ça va beaucoup plus vite mais la mise en place c'est très long et en fait là on a pas eu le temps vraiment de se poser, de réfléchir à quels moyens on va utiliser». A cette remarque font écho les propos d'une enseignante : «(...) on les met au travail mais nous non plus et eux non plus n'ont pas réfléchi à qu'est-ce qu'on utilise, comment on l'utilise et pourquoi (...)».

Pour les élèves cela fait finalement la marque de la vie lycéenne : «(...) c'est plus compliqué, ça demande une organisation »; «(...) on est beaucoup plus libres et c'est à nous de, nous-même, de poser des limites, à nous-même de nous organiser, de faire l'échéancier, (...) de gérer notre projet ». A ce titre, les réseaux sociaux et les outils collaboratifs du marché jouent donc un rôle central, en face-à-face et à distance : «Dès qu'il y a un projet quelconque au sein du lycée, où on est en petit groupes, c'est Facebook directement»; «C'est beaucoup plus pratique ». Ce recours systématique des élèves aux réseaux sociaux comme aux outils d'organisation «gratuits » proposés par des sociétés commerciales cohabite avec l'obligation qui leur est 
faite d'utiliser les outils du lycée. Ils passent de l'un à l'autre, non sans difficultés parfois. Cela a été montré par d'autres chercheurs (e.g. Lampe et al., 2011) et nous percevons ici une dimension importante mais cachée de leur travail, considérée par les élèves comme sans intérêt pour les enseignants : «Le serveur, c'est ce que les profs ont à aller regarder alors que Hangout c'est vraiment la mise en place ». Toute une dimension proprement organisationnelle demeure cantonnée dans ces usages considérés à la limite du personnel par les élèves, les enseignants n'ayant accès qu'à ce que les élèves considèrent comme digne d'être montré, évalué.

\section{Les outils mobilisés pour s'organiser et produire}

Outre l'obligation d'utiliser le réseau du lycée, les enseignants ne donnent pas d'instructions particulières sur les outils à utiliser. On peut noter par contre une influence significative des tactiques retenues en classe sur les habitudes quotidiennes des élèves : le recours systématique, par exemple, à une adresse Gmail lors de la réception des tablettes ou à Google Drive, issu d'habitudes prises en cours. Ainsi un enseignant explique : «(...) quand les tablettes sont arrivées de toute façon pour pouvoir faire une mise-à-jour sur Playstore, t'étais coincé. (...) On leur a fait créer des adresses Gmail génériques, (...) et d'ailleurs, ils se sont mis à utiliser beaucoup plus Gmail pour récupérer beaucoup de choses, à partir de ce moment-là. Et même si aujourd'hui il n'y a plus de tablette, enfin, de tablette sous Android, ils utilisent pour beaucoup encore Gmail ». Le recours systématique aux outils du marché (Google, Dropbox, Facebook) à côté des supports physiques (papier, clés USB, CD) et des outils recommandés ou créés par l'institution (l'ENT, le réseau informatique), pour s'organiser, stocker et produire, est déjà bien présent dans les choix d'organisation des élèves. Il l'est également dans les pratiques enseignantes et de façon assumée : « (...) c'est quand même des outils très efficaces, en termes de travail ». Si ces solutions font l'objet de réserves, quant à la protection des données personnelles en particulier, elles sont sollicitées pour leur efficacité et leur compatibilité avec les appareils mobiles omniprésents. Elles sont surtout en phase avec les pratiques sociales des élèves et des enseignants, et avec le besoin ressenti par les élèves de travailler en dehors.

Le recours aux outils du marché concerne toutes les étapes du dispositif pédagogique observé et qui font sa spécificité : la recherche de sources et d'informations, l'organisation collective et la production proprement dite. C'est un entrelacement complexe de pratiques sociales et scolaires auquel nous assistons : ces outils venant pragmatiquement au service des activités pédagogiques (en particulier pour le partage et le travail collectif), constituant aussi des objectifs d'apprentissage (éduquer les élèves à ces outils plutôt que de leur interdire de les utiliser) et prolongeant des habitudes quotidiennes qui ne sont ni scolaires ni professionnelles. Cette cohabitation ne s'effectue pas sans réserve, ainsi un enseignant évoque-t-il les arrangements entre efficacité pratique et objectifs pédagogiques : «On sait que ce n'est pas bien mais on le fait quand même parce que ça marche mais après on adapte avec les grilles d'évaluation ». 
De nature holistique, l'approche translittératique permet d'appréhender la richesse des pratiques en construction, lors des situations scolaires observées et en lien avec ce qui éventuellement les précède et/ou les prolonge. A ce titre, cette approche rejoint de multiples initiatives théoriques et méthodologiques s'attachant à décrire la complexité des pratiques distribuées, à la fois en ligne et hors ligne (Leander \& McKim, 2003 ; Pastinelli, 2011 ; Selwyn, 2010 ; Grosjean 2013 ; Donnat, 2017). La centration sur la seule dimension numérique de ces usages ferait ainsi perdre de vue les choix d'organisation individuels et collectifs, la combinaison des exigences scolaires et les tactiques ou stratégies mises en place pour y répondre, de la part des élèves comme de leurs enseignants. En outre, les compétences ici exigées par le lycée et que nous retrouvons aux niveaux national et international (travaux de groupe, recherche et traitement de l'information, création de contenus) ne reposent pas que sur la simple «maîtrise » des outils numériques, mais sur le choix d'outils compatibles avec ces attendus et avec les modalités très concrètes, parfois tâtonnantes, de leur mise en œuvre. Ce sont ces «espaces intermédiaires » tels que désignés par Cottier \& Burban, hybridant le scolaire et le hors espace-temps scolaire, qui sont occupés non seulement par les usages mais par les éléments constitutifs des compétences exigées (information, collaboration, participation). Ces compétences nécessitent en effet des outils adéquats et suffisamment fluides : à ce niveau, les outils du marché jouent un rôle prépondérant en même temps qu'en marge, en brouillon des productions finales qu'ils permettent cependant de réaliser. Pour les enseignants, cela revient dès lors à gérer une forme d'injonction paradoxale entre gage d'efficacité (recourir à des outils opérationnels et efficaces, compatibles avec les modes d'organisation collective et les objectifs scolaires) et enjeux éducatifs (connaître les modèles économiques et les politiques de ces plateformes en termes de gestion des données, par exemple). Il s'agit dès lors de, non seulement former à ces outils et à ce qu'ils sont, mais de comprendre ce qu'ils permettent de faire ou c'est le réel travail de l'élève qui peut dès lors se dérouler loin du regard enseignant. Cette situation n'est pas facile à gérer pour les enseignants, même a priori aguerris. Elle n'est de plus pas propre au lieu spécifique de notre investigation mais révèle un certain «vide pédagogique ». La question n'est pas simplement de dire que les outils de l'institution ne sont pas ou moins «efficaces » que les «solutions» du marché : ces outils, installés dans les pratiques quotidiennes, le sont aussi à l'école $a$ fortiori lorsque des domaines de compétences sont promus parmi lesquels la capacité à travailler en groupes et à prolonger le travail hors l'école. 
Amadieu, F. \& Tricot, A. (2014). Apprendre avec le numérique : Mythes et réalités. Retz.

Conein, B. (2004), Cognition distribuée, groupe social et technologie cognitive, Réseaux, 2(124), 53-79

Cottier, P. \& Burban, F. (Eds) (2016). Le Lycée en régime numérique : Usages et recomposition des acteurs.

Toulouse : Octares.

Delamotte, E., Liquète, V. \& Frau-Meigs, D. (2014). La translittératie, à la convergence des cultures de l'information : supports, contexte et modalités. Spirale, 53, 145-156.

Donnat, O. (dir) (2017). Les publics in situ et en ligne. Culture et Recherche, $\mathrm{n}^{\circ} 134$.

Grosjean, S. (2013). Une approche microethnographique et multi-située en organisation : Double mouvement de «zoom avant/arrière » sur l'activité d'arpentage. Revue internationale de psychosociologie et de gestion des comportements organisationnels, Supplément (HS), 155-177.

Ipri, T. (2010). Introducing Transliteracy: What does it mean to academic libraries? College and Research Libraries News, November: 532-567.

Jeanneret Y., Souchier E. et Le Marec J. (2003). Lire, écrire, récrire : objets, signes et pratiques des média informatisés. Paris, BPI.

Lampe, C., Wohn, D. Y., Vitak, J., \& Ellison, N. \& Wash, R. (2011). Student use of Facebook for organizing collaborative classroom activities. International Journal of Computer-Supported Collaborative Learning, 6(3), 329-347.

Leander, K.M. \& McKim, K. (2003). Tracing the Everyday 'Sitings' of Adolescents on the Internet: a strategic adaptation of ethnography across online and offline spaces. Education, Communication \& Information, 3(2), 212-240.

Leeder, C. \& Shah, C. (2016). Strategies, obstacles, and attitudes: student collaboration in information seeking and synthesis projects. Information Research, 21(3).

Liquète, V. (2012). Can one speak of an "Information Transliteracy"?. Paper presented at the Conference Media and Information Literacy for knowledge societies, Moscow https://hal.archives-ouvertes.fr/hal00841948/document

Pastinelli, M. (2011). Pour en finir avec l'ethnographie du virtuel ! Des enjeux méthodologiques de l'enquête de terrain en ligne. Anthropologie et Sociétés, 35(1-2), 35-52.

Robinson, L. (2015). Digital inequalities and why they matter. Information, Communication \& Society, $18(5), 569-582$.

Selwyn, N. (2010). Looking beyond learning: Notes towards the critical study of educational technology. Journal of Computer Assisted Learning, 26(1), 65-73.

Sukovic, S. (2017). Transliteracy in Complex Information Environments. Chandos Publishing.

Thomas, S, Joseph, C., Lacetti, J., Mason, B., Mills, S., Perril, S. \& Pullinger, K. (2007). Transliteracy: 